\title{
Two-Dimensional Correlation Analysis of Sum-Frequency Vibrational Spectra of Langmuir Monolayers
}

\author{
Jonggwan Lee, Woongmo Sung, and Doseok Kim* \\ Department of Physics, Sogang University, Seoul 121-742, Korea
}

(Received June 10, 2014 : revised August 4, 2014 : accepted August 19, 2014)

\begin{abstract}
Sum-frequency generation spectra of a Langmuir monolayer on water surface at varying surface areas were studied with two-dimensional correlation analysis. Upon enlarging the area/molecule of the Langmuir monolayer, the sum-frequency spectra changed reflecting the conformation change of the alkyl chains of the molecules in the monolayer. These changes stood out more clearly by employing two-dimensional correlation analysis of the above sum-frequency spectra. Features not very pronounced in the original spectra such as closely-spaced spectral bands can also be easily distinguished in the two-dimensional correlation spectra.
\end{abstract}

Keywords : Sum-frequency generation, 2D correlation analysis, Langmuir monolayer, Nonlinear optical spectroscopy, OPG/OPA

OCIS codes : (190.4350) Nonlinear optics at surfaces; (240.6490) Spectroscopy, surface; (300.6420) Spectroscopy, nonlinear

\section{INTRODUCTION}

Langmuir monolayers, which are monomolecular films of amphiphilic organic molecules at an air/water interface, have been investigated very extensively as a planar model system having a rich phase-transition behavior, and also as a simple system mimicking biological membranes [1-4]. Among the important issues concerning Langmuir monolayer are the conformation of molecules in the monolayer and its phase-transition behavior, and many experimental techniques such as X-ray diffraction/reflectivity $[5,6]$, Brewster angle microscopy [7, 8], ellipsometry [6, 8-11], and infrared reflection-absorption spectroscopy [9] have been employed to address these issues in the Langmuir monolayer system. As one of these techniques, sum-frequency generation (SFG) spectroscopy has proven to be the most promising and feasible technique to investigate the molecular conformation of the system $[10,12,13]$

Based on the second-order nonlinear optical process which is not allowed in the system having inversion symmetry, SFG is a highly surface-sensitive probe to any light-accessible interface. It is also suitable to monitor the molecular conformation and structure because the sum-frequency response of each vibrational mode is an orientation average of specific moieties of the molecules in the system and changes sensitively with a subtle conformation change of the molecules [14]. For example, when long-chain molecules in the monolayer are neatly ordered, the directions of methylene $\left(\mathrm{CH}_{2}\right)$ groups in each alkyl chain are oriented oppositely, to make the SF response from methylene groups cancel each other. Therefore, for a compact monolayer with molecules in all-trans conformation, the methylene group does not contribute to the signal, whereas terminal methyl groups having a net polar orientation only contribute to the SF spectrum [14]. On the other hand, when alkyl chains in the monolayer become disordered, methylene bands contribute significantly to the spectrum as compared to the case of all-trans conformation. Although each specific mode in the spectrum can be resolved by a fitting procedure, it is sometimes ambiguous to analyze sumfrequency spectra when the spectral features are not very pronounced. For example, at around $2950 \mathrm{~cm}^{-1}$, the spectral component of $\mathrm{CH}_{3}$ (methyl group) asymmetric stretch mode in alkyl chain is closely overlapped with other modes and the fitting often fails to resolve it from the other bands.

To better interpret the complex spectra, a two-dimensional

\footnotetext{
*Corresponding author: doseok@sogang.ac.kr

Color versions of one or more of the figures in this paper are available online.
} 
(2D) correlation analysis approach has been employed in IR and Raman spectroscopies [15]. By employing the mathematical formalism based on an integral transformation, this technique can help to follow the spectral changes caused by external perturbations more clearly. 2D correlation analysis outputs two two-dimensional arrays, called 2D synchronous and asynchronous maps, respectively. These maps work to enhance the spectral resolution by spreading one-dimensional data to two-dimensional arrays, and have been utilized to analyze many kinds of spectroscopic data in the system undergoing changes by an external perturbation [15]. When this technique was first developed in 1986 to enhance an ability to interpret complex spectra, it could be applied only to a time-dependent sinusoidal perturbation [16]. In the early 1990s its mathematical formalism was generalized based on Fourier transformation of the data, and gained a widespread usage [15]. Nowadays, a more advanced formalism based on Hilbert transformation is widely employed for calculation because of its computational efficiency and simplicity [17]. To date, 2D correlation analysis has been utilized to analyze many kinds of one-dimensional (1D) spectroscopic data including electronic, IR, NMR, X-ray and Raman spectra, and was found useful for an in-depth study of spectral variations caused by external factors [18-24]. Recently, Hore and coworkers first suggested applying $2 \mathrm{D}$ correlation analysis to investigate the simulated sum-frequency spectra, and demonstrated that 2D correlation analysis can also be readily applied to the sum-frequency vibrational spectroscopy [25].

In this work, 2D correlation analysis was first applied to the experimental SFG spectra. Langmuir monolayers containing the equal-amount mixture of two different kinds of lipid molecules was investigated at several different moleculararea conditions by sum-frequency generation spectroscopy. The change in the 1D spectra indicative of the conformation change of the molecules in the monolayer were shown more clearly in the 2D correlation maps [26]. Closely-spaced spectral bands in the $\mathrm{CH}_{\mathrm{x}}$ range (spectral range corresponding to the stretch modes of the alkyl chains) could be easily distinguished in the 2D correlation spectra. Sequential order of the spectral evolutions also came into sight from the asynchronous 2D correlation map. Some spectral components in correlation maps were found out to be shifted from the original position, due to the interference effect in the coherent spectroscopy.

\section{THEORY}

\subsection{Sum-Frequency Vibrational Spectroscopy}

A typical sum-frequency setup has two inputs of visible and IR beams, having frequencies of $\omega_{\mathrm{vis}}$ and $\omega_{\mathrm{TR}}$ to make it spatially overlapped at the sample surface. The output from the sample has a frequency of $\omega_{\mathrm{SFG}}=\omega_{\mathrm{is}}+\omega_{\mathrm{R}}$. According to the theory of surface sum-frequency generation, the intensity of SF output is determined as [12]:

$$
S(\omega) \propto\left|\vec{\chi}_{S}^{(2)}: E\left(\omega_{v i s}\right) E\left(\omega_{I R}\right)\right|^{2} .
$$

Here $\chi_{\mathrm{S}}^{(2)}$ is a second-order nonlinear susceptibility tensor, which can be written in terms of a second-order hyperpolarizability of the molecule $\alpha^{(2)}{ }_{\xi \eta \zeta}$ at the interface, and directional cosine between the laboratory $(\mathrm{x}, \mathrm{y}, \mathrm{z})$ and the molecular $(\xi, \eta, \zeta)$ axes as follow.

$$
\begin{aligned}
& \chi_{j k k}^{(2)}=N_{s} \sum_{\xi, \eta, \zeta}\langle(\hat{i} \cdot \hat{\xi})(\hat{j} \cdot \hat{\eta})(\hat{k} \cdot \hat{\zeta})) \alpha_{\xi \eta \zeta}^{(2)}, \\
& \alpha^{(2)}=\alpha_{N R}^{(2)}+\sum_{q} \frac{\alpha_{q}}{\omega_{I R}-\omega_{q}+i \Gamma_{q}} .
\end{aligned}
$$

Here $N_{\mathrm{S}}$ stands for the number density of molecules, $a_{q}$, $\omega_{q}$, and $\Gamma_{\mathrm{q}}$ represent peak strength, resonance frequency, linewidth constant for $q$-th vibrational mode, respectively. The sum-frequency response can get a resonance enhancement by tuning the IR input frequency to $\omega_{q}$, to yield spectroscopic information of the system.

From Eq. (2), SFG spectroscopy is especially useful to study subtle conformation changes in Langmuir monolayer system, which is a distinguishing feature as compared to the other probes like infrared absorption or Raman spectroscopy. If the chain has all-trans conformation, half of its methylene $\left(-\mathrm{CH}_{2}\right)$ groups have opposite direction to the other half, and their sum-frequency response cancels each other following Eq. (2). On the other hand, the methyl $\left(-\mathrm{CH}_{3}\right)$ group response is maximized for ordered monolayer, as all the molecules then have the same orientation. Disorder in the alkyl chain is going to change the symmetry of the system and the relative strength of these two bands, thus it can work as a sensitive indicator of the chain conformation.

\subsection{Two-dimensional Correlation Analysis}

Two-dimensional correlation analysis shows the spectral variation caused by an external perturbation as a pair of two-dimensional arrays, each of them called 2D synchronous and asynchronous spectrum of the system. Typical two-dimensional analysis approach follows several calculation steps. In this work, we only sketch the formalism based on Hilbert transformation. First, from a set of $m$ spectra taken by changing external conditions, one should get a dynamic spectrum by subtracting the average of all the spectra from each spectrum. Then synchronous spectrum $\Phi\left(v_{1}, v_{2}\right)$ is determined as:

$$
\Phi\left(v_{1}, v_{2}\right)=\frac{1}{m-1} \sum_{j=1}^{m} \tilde{y}_{j}\left(v_{1}\right) \tilde{y}_{j}\left(v_{2}\right)
$$

where index $j$ represents discrete evolution of external perturbation. Calculation of asynchronous spectrum $\Psi\left(v_{1}, v_{2}\right)$ 
is a little bit trickier:

$$
\begin{aligned}
& \Psi\left(v_{1}, v_{2}\right)=\frac{1}{m-1} \sum_{j=1}^{m} \tilde{y}_{j}\left(v_{1}\right) \tilde{z}_{j}\left(v_{2}\right) \\
& \text { where } \tilde{z}_{j}\left(v_{2}\right)=\sum_{k=1}^{m} N_{j k} \cdot \tilde{y}_{k}\left(v_{2}\right) \text { and } N_{j k}=\left\{\begin{array}{cc}
0 & \text { if } \mathrm{j}=\mathrm{k} \\
1 / \pi(k-j) & \text { otherwise }
\end{array}\right.
\end{aligned}
$$

Interpretation of these two-dimensional correlation spectra follows Noda's rule, named after Noda who first invented this mathematical formalism [27]. A peak in a synchronous 2D spectrum represents the frequency bands whose intensities vary in phase. Since every peak varies in phase with itself, a 2D synchronous spectrum always shows diagonal peaks. And there can be also off-diagonal peaks called cross peaks. If there is a positive cross-peak at $\left(v_{1}\right.$, $v_{2}$ ) in the synchronous spectrum, it implies that the intensities of the signals at $v_{1}$ and $v_{2}$ change simultaneously to the same direction. On the other hand, a negative cross peak at $\left(v_{1}, v_{2}\right)$ in the synchronous spectrum stands for a simultaneous change in opposite directions of the signals at $v_{1}$ and $v_{2}$. Asynchronous spectrum describes spectral changes which are temporally separated [27]. An asynchronous peak develops when the intensities of signal at different frequency bands vary out of phase. If a change in the band at $v_{1}$ takes place dominantly before a change in the band at $v_{2}$ the asynchronous cross peak at $\left(v_{1}, v_{2}\right)$ is positive. Conversely, a negative peak at $\left(v_{1}, v_{2}\right)$ implies that the change at $v_{1}$ follows the change at $v_{2}$.

As these 2D spectra are derived from a series of 1D spectra, these maps can determine the peak positions more accurately when two or more spectral modes are close together and cannot be resolved easily. It can also distinguish the processes that look very similar with the conventional 1D spectra. The case of (1) pure peak shift of single band, and (2) two overlapped peaks change in intensities under external perturbation sometimes cannot be distinguished easily in the 1D spectra. Dluhy and coworkers compared these two cases in IR absorption spectroscopy with 2D correlation analysis, and reported that a $2 \mathrm{D}$ asynchronous spectrum shows totally different appearance in the two cases. The former ('frequency shifting' model) shows a quartet of cross peaks, and the latter ('overlapped peaks' model) shows a doublet of cross peaks which is clearly different from the former one [28].

\section{EXPERIMENTAL SECTION \& DATA TREATMENT}

The 1,2-dipalmitoyl-3-trimethylammonium-propane (16:0 TAP or DPTAP) and 1,2-dimyristoyl-sn-glycero-3-phospho(1'-rac-glycerol) (14:0 PG or DMPG) molecules were used to build a monolayer. Both are purchased from Avanti

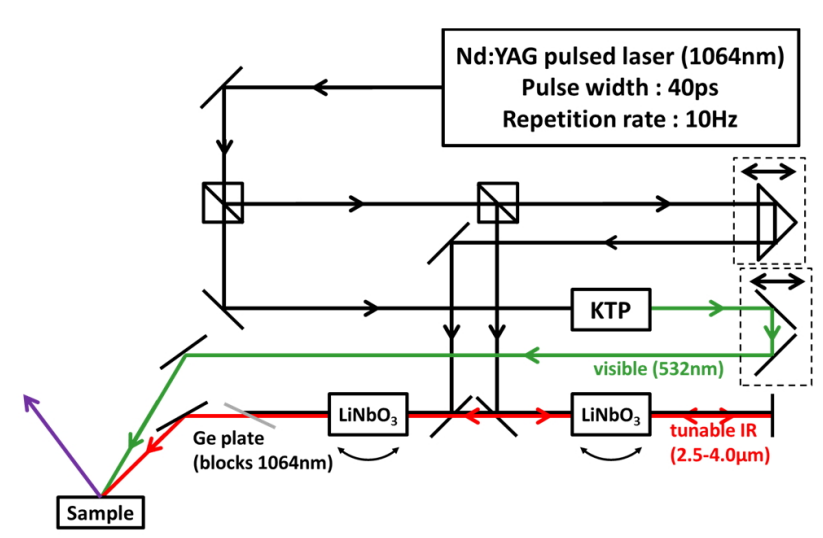

FIG. 1. Schematics of the SFG experimental setup.

Polar Lipids Inc. at $99+\%$ stated purity. Sample solution of lipids was prepared by dissolving in a pure chloroform solution ( $>99.9 \%$, HPLC grade, Aldrich) and spread onto a water subphase in a Langmuir trough [26]. The barriers of the trough were gently pushed or pulled to regulate the designated molecular area, and surface pressure of the monolayer was monitored simultaneously. The subphase was ultrapure water $(18.2 \mathrm{M} \Omega-\mathrm{cm})$ purchased from J. T. Baker.

The SFG measurement was conducted with a picosecond Nd:YAG pulsed laser (Continuum PY61-10, 40ps pulse width, $10 \mathrm{~Hz}$ repetition rate) and a $\mathrm{LiNbO}_{3}$-based optical parametric generator/amplifier (OPG/OPA) system pumped by this laser. The OPG/OPA system provided the tunable IR input ranging from $2.5 \mu \mathrm{m}$ to $4.0 \mu \mathrm{m}$ in wavelength, and the second-harmonic of the laser from potassium titanyl phosphate (KTP) crystal produced the visible input (532 $\mathrm{nm})$. Typical input energies were $1 \mathrm{~mJ} / \mathrm{pulse}$ for visible and $\sim 200 \mu \mathrm{J} / \mathrm{pulse}$ for tunable IR. Their incident angles were $\beta_{\mathrm{vis}}=45^{\circ}$ and $\beta_{\mathrm{IR}}=60^{\circ}$, respectively. These two beams were overlapped on the sample surface within the beam diameter of $\sim 200 \mu \mathrm{m}$. The SF output in the reflection direction was spatially and spectrally filtered and detected by a photomultiplier tube. The spectra were taken at typically $5 \mathrm{~cm}^{-1}$ intervals and each data point was an average of signals accumulated over at least 200 laser shots. The spectra were normalized against the SF spectrum from a z-cut quartz sample used as a reference. Input/output polarization combination of SSP (S-polarized SF output, S-polarized visible input and P-polarized IR input) was employed. 1D spectra were smoothed by averaging 3 adjacent data points as needed. Spectra were also normalized by dividing each spectrum with corresponding spectral area, as tabulated in Table 1. The array of the 1D spectra after normalization were treated with a lab-made MATLAB program for $2 \mathrm{D}$ correlation calculation, based on the generalized 2D correlation approach developed and modified by I. Noda. 


\section{RESULT \& DISCUSSION}

The SFG spectra of 1:1 mixture of DPTAP and DMPG Langmuir monolayers on water for five different surface areas, normalized by the total area under each spectrum, are plotted in Fig. 2. Here the monolayers with specific surface areas were prepared by compression of the monolayers with trough barrier, and regulated with an assistance of the pressure-area isotherm measured before [26]. Spectra show a gradual increase in $\mathrm{CH}_{2}$ symmetric stretch mode $\left(\sim 2853 \mathrm{~cm}^{-1}\right)$ and decrease in $\mathrm{CH}_{3}$ symmetric stretch mode $\left(\sim 2875 \mathrm{~cm}^{-1}\right)$ with an increase in the surface area, which implies a conformation change of monolayer from the condensed phase to the liquid phase. It would also cause the intensity changes in $\mathrm{CH}_{2}$ asymmetric, $\mathrm{CH}_{3}$ asymmetric stretch modes, and $\mathrm{CH}_{3}$ Fermi resonance mode (around $2919 \mathrm{~cm}^{-1}, 2959 \mathrm{~cm}^{-1}$ and $2936 \mathrm{~cm}^{-1}$, respectively), which can be hardly discriminated in the spectra because of their overlapping within narrow frequency band.

For a better discrimination of these features, two-dimensional correlation analysis was conducted on the spectra. Figure 3 shows a contour plot of 2D synchronous map (a) and a $2 \mathrm{D}$ asynchronous map (b) for the SF spectra normalized with spectral area shown in Fig. 2. In the synchronous map, a negative peak at $\left(2870 \mathrm{~cm}^{-1}, 2848 \mathrm{~cm}^{-1}\right)$ indicates an anti-correlation between $\mathrm{CH}_{2}$ and $\mathrm{CH}_{3}$ symmetric stretch modes clearly, which agrees with one-dimensional spectral analysis. Two-

dimensional correlation spectrum analysis shows its superiority in characterizing the closely-spaced spectral modes in

TABLE 1. Normalization factor for each spectrum

\begin{tabular}{c|c|c|c|c|c}
\hline \hline Molecular Area $\left(\AA^{2}\right)$ & 40 & 60 & 80 & 100 & 120 \\
\hline Spectral Area (a.u.) & 12.255 & 12.645 & 8.813 & 6.133 & 4.805 \\
\hline
\end{tabular}

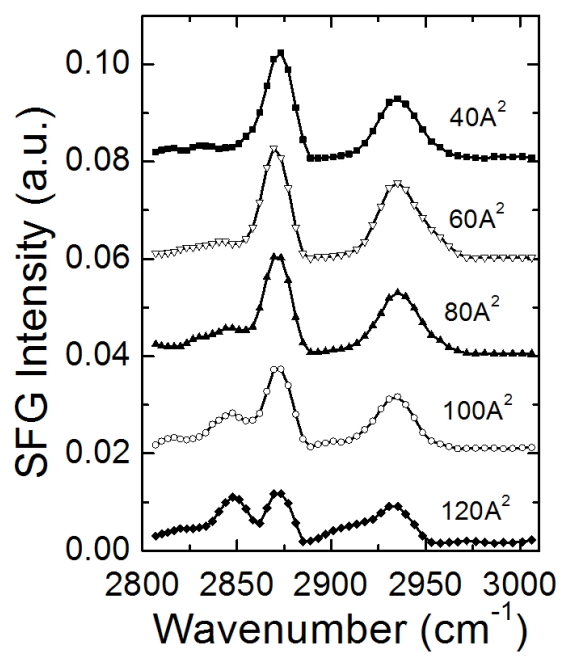

FIG. 2. SFG spectra of DPTAP/DMPG monolayers at different molecular areas at the surface. the region ranging from $2900 \mathrm{~cm}^{-1}$ to $2960 \mathrm{~cm}^{-1}$. Here one-dimensional analysis fails to distinguish different spectral components, whereas 2D synchronous map clearly shows the distinct negative and positive peaks. Negative peak at $\left(2870 \mathrm{~cm}^{-1}, 2910 \mathrm{~cm}^{-1}\right)$ implies the existence of a spectral component located around $2910 \mathrm{~cm}^{-1}$ negatively correlated with $\mathrm{CH}_{3}$ stretch mode at $2870 \mathrm{~cm}^{-1}$. This feature can be accounted for as the appearance of $\mathrm{CH}_{2}$ asymmetric stretch mode with an increased disorder in the alkyl chain. 2D synchronous map also shows a positive maximum at (2870 $\mathrm{cm}^{-1}, 2940 \mathrm{~cm}^{-1}$ ), suggesting the spectral feature positively correlated with $\mathrm{CH}_{3}$ symmetric stretch mode at $2870 \mathrm{~cm}^{-1}$. The peak is due to a decrease of $\mathrm{CH}_{3}$ Fermi resonance mode $\left(\sim 2936 \mathrm{~cm}^{-1}\right)$ simultaneous with a decrease of $\mathrm{CH}_{3}$ symmetric stretch mode as the molecular area increases. In the asynchronous spectrum in Fig. 3(b), the most prominent are the negative peaks located at $\left(2870 \mathrm{~cm}^{-1}, 2850 \mathrm{~cm}^{-1}\right)$ and $\left(2940 \mathrm{~cm}^{-1}, 2850 \mathrm{~cm}^{-1}\right)$. These asynchronous cross peaks indicate a different rate of change of these bands, and we deduce that the spectral change for $\mathrm{CH}_{2}$ mode $\left(\sim 2850 \mathrm{~cm}^{-1}\right)$ took place earlier than $\mathrm{CH}_{3}$ modes at 2870 $\mathrm{cm}^{-1}$ and $2940 \mathrm{~cm}^{-1}$.

One more thing to remark is that the locations of $2 \mathrm{D}$ spectral components are slightly shifted with respect to the conventional peak positions [14]. For example, in the

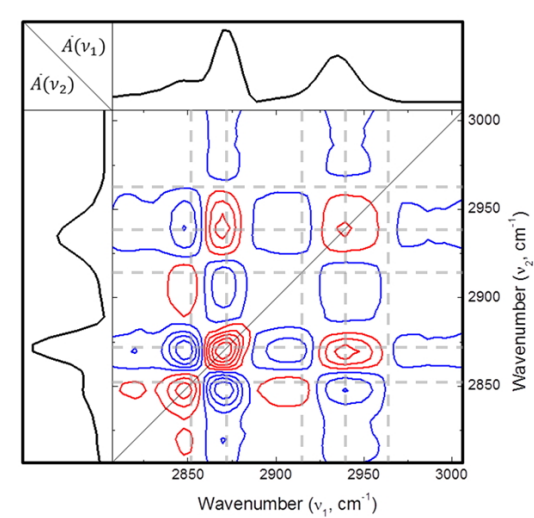

(a)

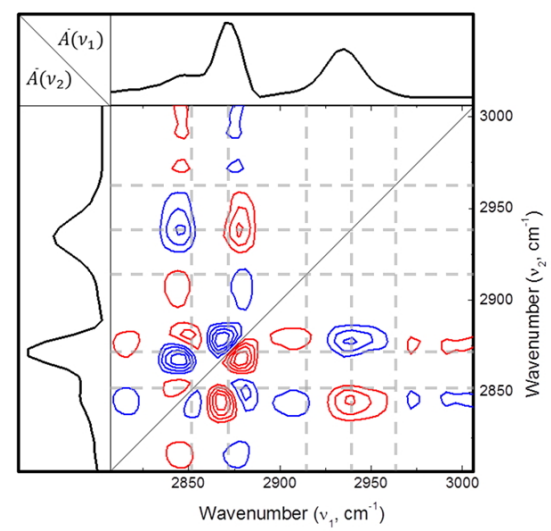

(b)

FIG. 3. 2D correlation maps of SF spectra of DPTAP DMPG monolayer. (a) Synchronous map, (b) Asynchronous map. 
synchronous map, the cross peak of $\mathrm{CH}_{2}$ symmetric stretch mode (conventionally about $2853 \mathrm{~cm}^{-1}$ ) and $\mathrm{CH}_{3}$ symmetric stretch mode $\left(\sim 2875 \mathrm{~cm}^{-1}\right)$ is located at $\left(2848 \mathrm{~cm}^{-1}, 2870 \mathrm{~cm}^{-1}\right)$, a few wavenumbers shifted from the original position. These kinds of spectral shifts also take place in the asynchronous map. These phenomena are considered as an artifact due to the intrinsic nature of the SFG spectroscopy. In conventional spectroscopy such as Raman, fluorescence, and IR absorption, the signals contributing to the spectral band (e.g. resonant signal and non-resonant background) are added incoherently as a spectral intensity. But in the sum-frequency generation spectroscopy, the signals contributing to a certain spectral feature are first added as electric fields. The field thus summed-up is squared to yield the SFG spectrum we measure experimentally, and the interference between spectral components can be seen in the spectrum when band-overlap or nonresonant contribution is significant. Recently S. Roy and coworkers reported that a spectral shift can be seen as an artifact when 2D correlation analysis is applied to the coherent spectroscopy [25]. To avoid this artifact, in the future study one can apply 2D correlation analysis to $1 \mathrm{D}$ imaginary spectra, which can be either deduced by fitting the spectrum with a multi-peak Lorentzian or measured with heterodyne SFG experiments [29].

\section{CONCLUSION}

Langmuir monolayers consisting 1:1 mixture of DPTAP and DMPG at different area/molecule were investigated with sum-frequency vibrational spectroscopy, and these spectra were further analyzed by using two-dimensional correlation spectroscopy. 2D correlation maps were able to better resolve closely-spaced spectral components, for example $\mathrm{CH}_{2}$ asymmetric stretch mode and $\mathrm{CH}_{3}$ Fermi resonance mode of the spectra. The $2 \mathrm{D}$ asynchronous map indicated the order of spectral evolution that the change in the $\mathrm{CH}_{2}$ mode precedes the change in the $\mathrm{CH}_{3}$ mode. Due to the interference between the bands in the SF spectra, spectral features in the correlation maps are found to be shifted from their originally assigned locations, which indicate that the care needs to be taken in analyzing the coherent $1 \mathrm{D}$ spectra by using the $2 \mathrm{D}$ correlation method.

\section{ACKNOWLEDGMENT}

This work was supported by the National Research Foundation (NRF) grant funded by the Korea Government (MEST) Nos. 2011-0017435 and 2011-0031496.

\section{REFERENCES}

1. H. Brockman, "Lipid monolayers: Why use half a membrane to characterize protein-membrane interactions?," Curr. Opin.
Struct. Biol. 9, 438-443 (1999).

2. M. Montal and P. Mueller, "Formation of bimolecular membranes from lipid monolayers and a study of their electrical properties," Proc. Natl. Acad. Sci. USA 69, 3561-3566 (1972).

3. F. Giess, M. G. Friedrich, J. Heberle, R. L. Naumann, and W. Knoll, "The protein-tethered lipid bilayer: A novel mimic of the biological membrane," Biophys. J. 87, 3213-3220 (2004).

4. M. Tanaka and E. Sackmann, "Polymer-supported membranes as models of the cell surface," Nature 437, 656-663 (2005).

5. M. Shih, T. Bohanon, J. Mikrut, P. Zschack, and P. Dutta, "X-ray-diffraction study of the superliquid region of the phase diagram of a Langmuir monolayer," Phys. Rev. A 45, 5734-5737 (1992).

6. S. R. Wasserman, G. M. Whitesides, I. M. Tidswell, B. M. Ocko, P. S. Pershan, and J. D. Axe, "The structure of self-assembled monolayers of Alkylsiloxanes on silicon: A comparison of results from ellipsometry and low-angle x-ray reflectivity," J. Am. Chem. Soc. 111, 5852-5861 (1989).

7. E. Loste, E. D.-Marti, A. Zarbakhsh, and F. C. Meldrum, "Study of calcium carbonate precipitation under a series of fatty acid Langmuir monolayers using brewster angle microscopy," Langmuir 19, 2830-2837 (2003).

8. Q. Huo, S. Russev, T. Hasegawa, J. Nishijo, J. Umemura, G. Puccetti, K. C. Russell, and R. M. Leblanc, "A Langmuir monolayer with a nontraditional molecular architecture," J. Am. Chem. Soc. 122, 7890-7897 (2000).

9. J. Gun, R. Iscovici, and J. Sagiv, "On the formation and structure of self-assembling monolayers: II. A comparative study of Langmuir-Blodgett and adsorbed films using ellipsometry and IR reflection-absorption spectroscopy," J. Colloid Interface Sci. 101, 201-213 (1984).

10. P. G.-Sionnest, J. H. Hunt, and Y. R. Shen, "Sum-frequency vibrational spectroscopy of a Langmuir film: Study of molecular orientation of a two-dimensional system," Phys. Rev. Lett. 59, 1597-1600 (1987).

11. S. Seok, T. J. Kim, S. Y. Hwang, Y. D. Kim, D. Vaknin, and D. Kim, "Imaging of collapsed fatty acid films at air-water interfaces," Langmuir 25, 9262-9269 (2009).

12. W. Sung, D. Kim, and Y. R. Shen, "Sum-frequency vibrational spectroscopic studies of Langmuir monolayers," Curr. Appl. Phys. 13, 619-632 (2013).

13. W. Sung, D. Vaknin, and D. Kim, "Different adsorption behavior of rare earth and metallic ion complexes on Langmuir monolayers probed by sum-frequency generation spectroscopy," J. Opt. Soc. Korea 17, 10-15 (2013).

14. X. Zhuang, P. B. Miranda, D. Kim, and Y. R. Shen, "Mapping molecular orientation and conformation at interfaces by surface nonlinear optics," Phys. Rev. B 59, 12632-12640 (1999).

15. I. Noda, "Generalized two-dimensional correlation method applicable to infrared, Raman, and other types of spectroscopy," Appl. Spectrosc. 47, 1329-1336 (1993).

16. I. Noda, "Two-dimensional infrared spectroscopy," J. Am. Chem. Soc. 111, 8116-8118 (1989).

17. I. Noda, "Determination of two-dimensional correlation spectra using the Hilbert transform," Appl. Spectrosc. 54, 994-999 (2000). 
18. M. Kačuráková, A. C. Smith, M. J. Gidley, and R. H. Wilson, "Molecular interactions in bacterial cellulose composites studied by 1D FT-IR and dynamic 2D FT-IR spectroscopy," Carbohydr. Res. 337, 1145-1153 (2002).

19. Q.-X. Ruan and P. Zhou, "Sodium ion effect on silk fibroin conformation characterized by solid-state NMR and generalized 2D NMR-NMR correlation," J. Mol. Sturct. 883-884, 85-90 (2008).

20. V. A. Shasilov and I. K. Lednev, "2D correlation deep UV resonance raman spectroscopy of early events of lysozyme fibrillation: Kinetic mechanism and potential interpretation pitfalls," J. Am. Chem. Soc. 130, 309-317 (2008).

21. I. Noda, Y. Liu, and Y. Ozaki, "Two-dimensional correlation spectroscopy study of temperature-dependent spectral variations of N-Methylacetamide in the pure liquid state. 2. Twodimensional Raman and infrared-Raman heterospectral analysis," J. Phys. Chem. 100, $8674-8680$ (1996).

22. Y. M. Jung, B. C.-Matusewicz, and Y. Ozaki, "Two-dimensional infrared, two-dimensional Raman, and two-dimensional infrared and Raman heterospectral correlation studies of secondary structure of â-Lactoglobulin in buffer solutions," J. Phys. Chem. B 104, 7812-7817 (2000).

23. Y. M. Jung, H. S. Shin, B. C. Matusewicz, I. Noda, and S. B. Kim, "Characterization of transition temperatures of a Langmuir-Blodgett film of Poly(tert-butyl methacrylate) by two-dimensional correlation spectroscopy and principal component analysis," Appl. Spectrosc. 56, 1568-1574 (2002).
24. H. C. Choi, Y. M. Jung, I. Noda, and S. B. Kim, "A study of the mechanism of the electrochemical reaction of lithium with $\mathrm{CoO}$ by two-dimensional soft x-ray absorption spectroscopy (2D XAS), 2D Raman, and 2D heterospectral XAS-Raman correlation analysis," J. Phys. Chem. B 107, 5806-5811 (2003).

25. S. Roy, J. S. Post, K.-K. Hung, U. Stege, and D. K. Hore, "2D correlation analysis in vibrational sum-frequency generation spectroscopy,” J. Mol. Struct. 1069, 103-111 (2014).

26. W. Sung, S. Seok, D. Kim, C. Tian, and Y. R. Shen, "Sum-frequency spectroscopic study of Langmuir monolayers of lipids having oppositely charged headgroups," Langmuir 26, 18266-18272 (2010).

27. I. Noda and Y. Ozaki, Two-Dimensional Correlation Spectroscopy-Applications in Vibrational and Optical Spectroscopy (John Wiley \& Sons, Chichester, UK, 2004).

28. D. L. Elmore and R. A. Dluhy, "Application of 2D IR correlation analysis to phase transitions in Langmuir monolayer films," Colloids Surf. A 171, 225-239 (2000).

29. I. V. Stiopkin, H. D. Jayathilake, A. N. Bordenyuk, and A. V. Benderskii, "Heterodyne-detected vibrational sum frequency generation spectroscopy," J. Am. Chem. Soc. 130, 22712275 (2008). 